\title{
ENERGY ANALYSIS OF DIFFERENT TRUNK TILTING ANGLES ON JOINT LOADING DURING FALL ON AN OUTSTRETCHED HAND
}

\author{
Ming-Chang Tsai' , Pei-Hsi Chou ${ }^{2}$,You-Li $\mathrm{ChOU}^{3}$, Ting-Sheng Lin ${ }^{4}$ \\ 'Mechanical Engineering, National Kaohsiung University of Applied Sciences, Kaohsiung \\ ${ }^{2}$ Department of Orthopedic Surgery, Kaohsiung Medical University, Kaohsiung \\ ${ }^{3}$ Center of General Education, Chang Jung Christian University, Tainan \\ ${ }^{4}$ Institute of Biomedical Engineering. National Cheng Kung University, Tainan, Taiwan
}

\section{ABSTRACT}

From previous researches, studies on the forward fall had focused on the relationship between the joint loading and position of the forearm and elbow. Previous studies also stressed the importance of energy absorption by the shoulder and elbow during the forward fall. However, the effects of different tilting angles on joint loading of the upper extremity had not been presented. This study investigated the effects of different tilting angles on joint loading of the upper extremity during fall on an outstretched hand. The absorbed energy at Ts, TI, T2 and Te were analyzed. Ten healthy young males were selected in this study. Subjects were adjusted to different trunk tilting angles of $0^{\circ}$. $10^{\circ} .20^{\circ}$ and $30^{\circ}$ with a custom-made suspension system with an outstretched hand of $5 \mathrm{~cm}$ height above the ground. The expert vision motion system with 6 CCD cameras and one force plate were used to collect kinetics and kinematics data. The results showed that shoulder would absorb the most impact energy and followed by wrist and elbow. The time period at T2 to Te absorbed more energy in every joint.

Biomed Eng Appl Basis Comm, 2003 (December); 15: 217-222.

\section{INTRODUCTION}

When a fall occurred, people often used upper extremities to protect the head and trunk from impact injuries[8,11,16]. Falling onto the outstretched hand was the major cause of upper extremity injury, including approximately $90 \%$ of fractures at the distal radius, humeral neck, and supracondyles of the

Received: Sept 25, 2003; Accepted: Nov 6, 2003

Correspondence: You-Li Chou, Ph.D., Professor

Center of General Education, Chang Jung Christian

University, Tainan, Taiwan

E-mail: ylchou@mail.ncku.edu.tw elbow $[9,13]$. The fall-related upper extremity fractures have a bimodal age distribution in young males and older females $[3,12]$. Robinovch and $C h i u[1]$ measured impact forces during forward fall onto the outstretched hand. From the reports, ground reaction forces are governed by an initial high-frequency peak (Fmax I) and followed by a low-frequency oscillation (Fmax2). They consider this behavior could be demonstrated using a two degree of freedom lumped parameter mathematical model to simulate falls on the outstretched hand. Neglecting the elbow motion, this mathematical model predicted that a fall from over $0.6 \mathrm{~m}$ heights would tesult in higher risk for wrist fracture. 
Other study revealed that the fall arrest strategy would affect peak hand impact force in a forward fall[4]. As reported by Chou et al.[2], elbow flexion, instead of outstretch hand, during a fall would decrease the ground reaction force effectively. They suggested that the motion of elbow flexion afforded the similar function of the combination of damper and spring. However, falling with truck tilting might be most popular way in daily activities and seldom studies were mentioned about this case. The purpose of this study is to investigate the joint force and moment when trunk was tilting during a fall. Four different trunk tilting angles, including $0^{\circ}, 10^{\circ}, 20^{\circ}$ and $30^{\circ}$, were applied in this study.

\section{METHODS}

\subsection{Subjects and Experimental Protocol}

Ten male subjects were included in this study. The basic data of subjects were ranged from 16 to 20 years (mean 18.0, SD 2.0) of age, 62 to $83 \mathrm{~kg}$ (mean 71.1 , SD 6.9) of body weight, and 165 to $183 \mathrm{~cm}$ (mean 175.4, SD 6.0) of body height. All subjects were right hand dominant without musculoskeletal disorder of the upper extremity.

The Expert Vision motion system (Motion Analysis Corp., Santa Rosa, CA, USA), consist of six cameras $(120 \mathrm{~Hz})$ and one force-plate with $1000 \mathrm{~Hz}$ sampling rate (Type 9281B, Kistler Instrument Corp., Winterthur, Switzerland), were used to record relative joint positions and ground reaction forces. A set of eleven reflective markers was placed on selected anatomic landmarks on the subject (as shown in Figure 1). These anatomic landmarks were assumed as rigid body and without relative motion between them. These markers included trunk (cervical vertebra 7 , thoracic vertebra 4 and acromion), upper arm (acromion process, medial and lateral epicondyles of the elbow), forearm (medial and lateral epicondyles of the elbow, ulnar styloid processes), and hand (radial and ulnar styloid processes, third metacarpal bone). In addition, a triangular frame with three-markers was placed on the upper arm in order to minimize the possible errors due to skin movement of epicondyles during experiments.

In order to find out the upper extremity joints energy absorption with different trunk tilting angles during a fall, all subjects were tested for four different trunk tilting angles $\left(0^{\circ}, 10^{\circ}, 20^{\circ}\right.$ and $\left.30^{\circ}\right)$. In the beginning, the subject was placed with knees in contact with the ground. Then the subject was suspended with a custom-made suspension system and fell from a height of $5 \mathrm{~cm}$ (i.e. distance between the outstretched hand and the force plate) with one hand. The angle between the trunk and the upper arm is maintained at

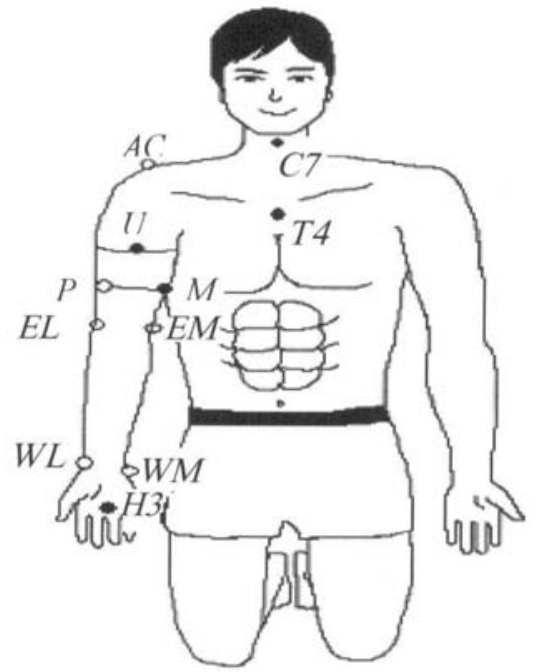

Fig. 1 Custom-made suspension system ('O'show forward markers and ' $O$ ' show forward markers. T4: $4^{\text {th }}$ thoracic vertebra. $\mathrm{C} 7: 7^{\text {th }}$ cervical vertebra. AC : Acromion. EL : lateral epicondyle of the humerus. EM : medial epicondyle of the humerus. WL : styloid process of radius. WM : styloid process of ulna. $\mathrm{H3}: 3^{\text {rd }}$ metacarpal bone. $U \cdot P \cdot M:$ marker triad)

$60^{\circ}$ forward flexion of the shoulder (Figure 2). Finally, the subject was tested with an outstretched hand following the procedures mentioned above. Furthermore, a questionnaire was also designed to record each subject's sensation of pain or discomfort index (ranged from 0 to 10) during the impact. High value of the index represented most painful or discomfort.

\subsection{Assumptions}

Three assumptions were made in this experiment: (1) each segment of upper extremity was assumed as rigid body with constant density; (2) each joint was assumed to have three rotational degrees of freedom; (3) all frictions, including air and rope/pulley (of the safety restraint system), were neglected.

\subsection{Theorem and Govern Equation}

Theorem of this study is based on Newton's second law of motion, and the free body diagram of each segment as shown in Figure 3.

Joint force : $\bar{F}_{p}+\bar{F}_{\nu}+m \bar{g}=m \bar{a}$

Joint moment $: \bar{M}_{p}+\bar{M}_{a}+\bar{r}_{p} \times \bar{F}_{p}+\bar{r}_{d} \times \bar{F}_{d}+\bar{\omega} \times(I \bar{\omega})=I \bar{\alpha}$

Joint power : $P(t)=\bar{M}(t) \cdot d \bar{\omega}(t)=\bar{M}(t) \frac{d \vec{\theta}(t)}{d t}$ 
Joint energy $: \int \vec{M}(t) \cdot \frac{d \bar{\theta}(t)}{d t} d t=\int \vec{M}(t) \cdot d \vec{\theta}(t)$

The symbols of the equilibrium equations are explained as below:

$\bar{F}_{p}$ : proximal joint force

$\bar{F}_{a}$ : distal joint force.

$m \vec{g}:$ gravity force of the local segment.

$m \bar{a}:$ effective force.

$\bar{M}_{\odot}$ : proximal joint moment.

$\bar{M}_{\triangleleft}:$ distal joint moment.

$\bar{r}_{.}$: position vector of the distal end moment arm of the segment.

$\bar{r}_{p}$ : position vector of the proximal end moment arm of the segment.

$\bar{\omega}:$ angular velocity of the local segment.

$\vec{\alpha}$ : angular acceleration of the local segment.

$I$ : mass moment of inertia.

\subsection{Data Reduction}

Laboratory-developed kinematics and kinetics software were used to calculate the joint angles, resultant forces and moments of the wrist, elbow and shoulder. A three-segment model, i.e. hand, forearm and upperarm, was employed in the analysis. Three joints angles, hinge angle, rotational angle and horizontal deviation were calculated using Euler's method with a $y-x-z$ rotational sequence based on the attached markers. Segment mass and inertia data were estimated by human anthropometry[5]. Angular velocity and acceleration were calculated with Euler's

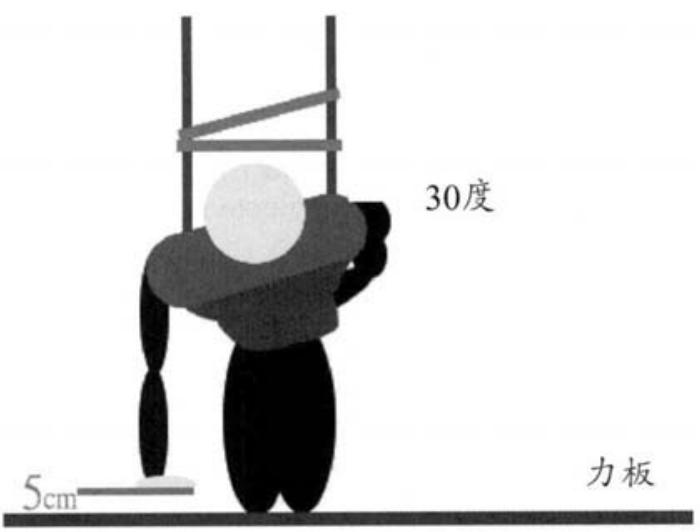

Fig. 2 The experimental arrangement.

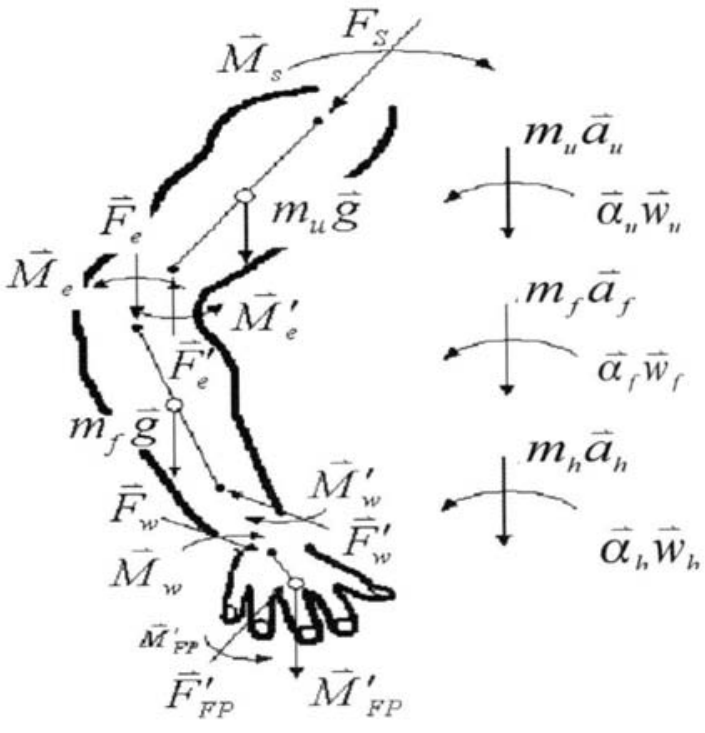

Fig. 3 The free body diagram of upper extremity.

parametric method [7]. The joint loadings were then obtained using inverse dynamic procedures with the Newton-Euler equations. A generalized crossvalidation spline smoothing (GCVSPL) routine with a cutoff frequency of $6 \mathrm{~Hz}$ was used for data smoothing [15]. Joint angles and joint forces of the wrist, elbow and shoulder as functions of time during the impact period were calculated and then used for analysis.

\subsection{Data Analysis}

Statistical analyses (ANOVA), with statistical significance set at the $5 \%$ level, were used to calculate for the ground reaction force, joint angle and joints force. In order to evaluate the vertical force data of the ground reaction force (GRF), the entire time period of each single test was divided into three time phases: (1) the Pre-Impact phase, lasting from $\mathrm{T}_{0}$ to $\mathrm{Ts}$; (2) the Impact phase, lasting from Ts to Te; (3) the PostImpact phase, lasting from Te to the end of the experiment. To was the instant when the fall began. Ts was the instant when the ground reaction force (GRF) rose above baseline noise level. Te was defined as the end of the final rapid decrease in GRF prior to the steady state response (Figure 4).

\section{RESULT}

\subsection{Energy Absorption and Joint Energy}

Energy absorption was defined as elbow joint energy divided by shoulder joint energy (Elbow/Shoulder) and wrist joint energy divide by shoulder joint energy (Shoulder/Wrist). The energy 


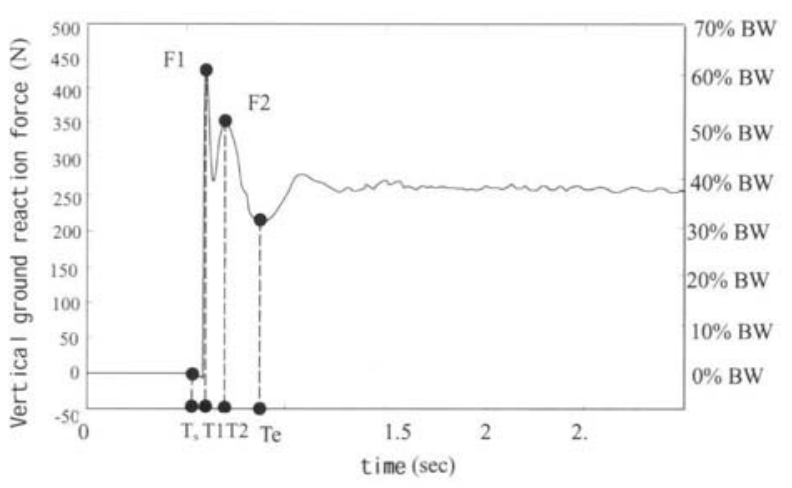

Fig. 4 Typical GRF data of neutral posture.

absorption from Ts to Te period among four groups were not significantly different $(\mathrm{P}<0.05)$ (Table 1 ). During Ts toTl period, the impact energy among wrist, elbow and shoulder joint were smaller than other period, and there were not significantly different among four groups. During Ts toT2 period, the value was also not significantly different among four groups.
During Ts to Te period, the value was increased obviously, but there was no significant difference among four groups. The period from T2 to $\mathrm{Te}$ is the mean period for joint to absorb impact energy during falls. Even wrist, elbow or shoulder joint energies were increased obviously in this period, there was no significant difference among four groups in every time period (Table 3). The results of questionnaire showed that $0^{\circ}$ group has the lower uncomfortable index (Table 4).

\section{DISCUSSION}

During falls, the impact energy would be absorbed by upper extremity joints' motion as the effect of damper. In this study the subjects were requested to be completely outstretched their elbow in order to protect the direct impact to body and head. However, stronger impact force resulted in fewer elbow angle motion that may be affected the energy absorption in elbow joint. Wrist joint had absorbed energy by forearm muscle contraction and valgus motion. Shoulder absorbed energy by back muscle and forearm muscle contraction.

Table 1. Total energy absorptance for each different group.

\begin{tabular}{|l|c|c|c|c|}
\hline & 0 degree & 10 degree & 20 degree & 30 degree \\
\hline (Shoulder/Elbow) & $5.49(0.64)$ & $5.12(0.71)$ & $5.37(0.68)$ & $5.25(0.83)$ \\
\hline (Shoulder/Wrist) & $2.91(0.18)$ & $2.73(0.23)$ & $2.49(0.12)$ & $3.05(0.31)$ \\
\hline
\end{tabular}

Table 2. Joint energy ( $\mathrm{N}-\mathrm{m})$ during different period.

\begin{tabular}{lcccc}
\hline & $0^{\circ}$ & $10^{\circ}$ & $20^{\circ}$ & $30^{\circ}$ \\
\hline wrist joint energy & & & & \\
Ts to T1 period & $1.5(0.3)$ & $1.3(0.4)$ & $1.8(0.4)$ & $1.7(0.4)$ \\
Ts to T2 period & $7.3(3.0)$ & $8.4(3.1)$ & $8.1(2.9)$ & $8.9(3.0)$ \\
Ts to Te period & $16.0(5.7)$ & $17.8(5.6)$ & $18.2(5.1)$ & $16.9(5.9)$ \\
elbow joint energy & & & & \\
Ts to T1 period & $0.9(0.0)$ & $1.1(0.1)$ & $1.0(0.2)$ & $1.1(0.2)$ \\
$T_{s}$ to $T_{2}$ period & $3.9(0.9)$ & $4.2(0.9)$ & $4.0(1.0)$ & $4.3(0.9)$ \\
Ts to Te period & $9.4(2.7)$ & $9.1(2.4)$ & $10.2(3.3)$ & $9.9(3.1)$ \\
shoulder joint energy & & & & \\
Ts to T1 period & $2.5(0.6)$ & $2.7(0.8)$ & $2.1(0.6)$ & $3.0(0.5)$ \\
Ts to T2 period & $28.4(5.4)$ & $30.4(6.1)$ & $28.0(5.2)$ & $31.1(6.0)$ \\
Ts to Te period & $46.7(6.7)$ & $48.7(6.4)$ & $45.4(6.4)$ & $50.7(6.0)$ \\
\hline
\end{tabular}

Unit : N-m "** express $\mathbf{p}<0.05$ 
Table 3. Percentage of joint energy during different period.

\begin{tabular}{|c|c|c|c|c|c|}
\hline & $0^{\circ}$ & 10 & $0^{\circ}$ & $20^{\circ}$ & $30^{\circ}$ \\
\hline \multicolumn{6}{|l|}{ wrist joint } \\
\hline$T_{s}$ to $T_{1}$ period & $2.1(0.9)$ & $1.7(0.5)$ & $2.4(0.8)$ & $2.2(0.9)$ & \\
\hline$T_{1}$ to $T_{2}$ period & $7 . .9(1.8)$ & $9.4(2.2)$ & $8.6(1.6)$ & $9.2(2.0)$ & \\
\hline $\begin{array}{l}T_{2} \text { to } T_{e} \text { period } \\
\text { elbow joint }\end{array}$ & $12.2(3.5)$ & $12.5(3.8)$ & $13.7(3.7)$ & $10.2(3.1)$ & \\
\hline$T_{s}$ to $T_{1}$ period & $0.2(0.0)$ & $2.0(0.7)$ & $1.4(0.4)$ & $1.4(0.4)$ & \\
\hline$T_{1}$ to $T_{2}$ period & $4.1(1.4)$ & $4.1(1.7)$ & $4.0(1.2)$ & $4.0(1.6)$ & \\
\hline $\begin{array}{l}T_{2} \text { to } T_{e} \text { period } \\
\text { shoulder joint }\end{array}$ & $7.5(1.9)$ & $6.5(2.0)$ & $8.4(2.4)$ & $7.1(2.0)$ & \\
\hline$T_{s}$ to $T_{1}$ period & $3.5(1.3)$ & $3.52(1.1)$ & $2.9(1.0)$ & $3.9(1.4)$ & \\
\hline$T_{1}$ to $T_{2}$ period & $35.9(5.7)$ & $36.6(5.2)$ & $35.0(5.2)$ & $35.7(5.8)$ & \\
\hline$T_{2}$ to $T_{e}$ period & $25.4(3.7)$ & $24.2(3.8)$ & $23.5(3.1)$ & $26.2(4.4)$ & \\
\hline
\end{tabular}

Table 4. Discomfort index.

\begin{tabular}{|l|cccc|}
\hline groups & 0 degree & 10 degree & 20 degree & 30 degree \\
\hline Uncomfortable index & 4.9 & 5.4 & 5.7 & 6.3 \\
\hline
\end{tabular}

\section{REFERENCES}

From the results of energy absorption, the shoulder joint would absorb the most of impact energy. Elbow joint absorbed much less energy than shoulder or wrist. During experiments process, the subject's knee have contacted the ground and absorbed some impact energy. Therefore, upper extremity joints were not significant difference among four groups in energy absorption and joint energy.

\section{CONCLUSION}

The experiments of different trunk tilting angles with $5 \mathrm{~cm}$ fall height during fall on an outstretched hand were carried out in this study. The results showed that shoulder would absorb the most impact energy and followed by wrist and elbow. The time period at T2 to Te absorbed more energy in every joint.
1. Chiu j., Robinovitch S.N., Prediction of upper extremity impact forces during falls on the outstretched hand. Journal of Biomechanics; 31(12): 1169-76, (1998).

2.Chou P.H., Chou Y.L., Lou S.Z., Lin C.J., Chiu C.F., Su F.C., Effect of elbow flexion on impact forces of upper extremity on a fall. Clinical Biomechanics 16,888-894, (2001).

3. Cummings, S.R., Klingberg, R.J., Fall frequency and characteristics and the risk of hip fractures. Journal of American Geriatrics Society 42, 774-778.

4. Degoede, K.M., Ashton-Miller, J.A., Fall arrest strategy affects peak hand impact force in a forward fall Journal of Biomechanics 35:843-848, (2002).

5 . Dempster WT. Space requirements of the seated operator. OH: Wright-Patterson Air Force Base, Dayton, 1955: 55-159.

6. Grisso, J.A., Kelsey, J.L., Strom, B.L. et al., Risk factors for falls as a cause of hip fracture in women. New England Journal of Medicine 324, 1326-1331, (1991). 
7. Haug EJ. Computer Aided Kinematics and Dynamics of Mechanical Systems Volume I: Basic Methods. Massachusetts: Allyn and Bacon, (1989).

8. Hsiao. E.T. Robinovitch. S.N. Common protective movements govern unexpected falls from standing height. Journal of Biomechanics 31.1-9, (1998).

9. Melton L.J., Chao E.Y.S., Lane j., Biomechanical aspects of fractures. In: Riggs B.L., Melton L.J.(Eds).Osteoporosis: Etiology, Diagnosis, and Management. Raven Press. New York,111-131, (1988).

10.Morrey B.F.,An K.N., Storronont T.J.,Force tramsmission throught the radial head. Journal of Bone and Joint Surgery 1988; 70A: 250-256.

11. O'Neill, T.W., Varlow, J., Silman, A.J., Reeve.J, Reid, D.M, Todd. C., Woolf, A.D. Age and sex influences on fall characteristics. Annals of Rheumatic Diseases 53,773-775, (1994).
12. Palvanen, M, Kannus, P, Parkkari, J. Pitkajarvi, T, Pasanen, M, Vuori, fractures among older adults: a controlled study of consecutive patients and their controls. Osteoporos International 1, 822-831, (2000).

13. Rockwood C.A., Jr., Wilkens K.E., King R.E., Fractures in Children. J.B. Lippencott, Philadephia,P.A, (1987).

14. Spaite,D.W., Criss, E.A., Valenzuela, T.D., Meislin, H.W., Ross, J., Geriatric injury: an analysis of prehospital demographics, mechanisms, and patterns. Annals of Emergency Medicine 19, 1418$1421,(1990)$.

15.Woltring H.J., A FORTRAN package for generalized, cross-validatory spline smoothing and differentiation. Advanced Engineering Software 1986; (8): 104-13.

16. Vellas, B.J. Wayne. S.J., Garry, P.J. Baumgartner, R, N.A two-year longitudinal study of falls in community-dwelling elderly adults. Journal of Gerontology 53A, M264-M274, (1998). 